\title{
Dietary intake assessment in women with different weight and pregnancy status using a short questionnaire
}

\author{
Åsa Svensson ${ }^{1, *}+$, Frida Renström ${ }^{2,3}$, Les Bluck ${ }^{4}$, Lauren Lissner ${ }^{5}$, Paul W Franks ${ }^{2,3,6}$ \\ and Christel Larsson ${ }^{1,7}$ \\ ${ }^{1}$ Department of Food and Nutrition, Umeå University, Umeå, Sweden: ${ }^{2}$ Genetic and Molecular Epidemiology \\ Unit, Department of Clinical Sciences, Lund University, Malmö, Sweden: ${ }^{3}$ Department of Public Health and \\ Clinical Medicine, Umeå University, Umeå, Sweden: ${ }^{4}$ MRC Human Nutrition Research, Cambridge, UK: \\ ${ }^{5}$ Department of Public Health and Community Medicine, Public Health Epidemiology Unit, Sahlgrenska \\ Academy, University of Gothenburg, Gothenburg, Sweden: ${ }^{6}$ Department of Nutrition, Harvard School of Public \\ Health, Boston, MA, USA: 'Department of Food and Nutrition, and Sport Science, University of Gothenburg, \\ Gothenburg, Sweden
}

Submitted 8 February 2013: Final revision received 7 August 2013: Accepted 15 October 2013: First published online 15 November 2013

\begin{abstract}
Objective: First, to evaluate the ability of a short dietary questionnaire (SDQ) to estimate energy intake (EI) on group and individual levels compared with total energy expenditure (TEE) measured by the doubly labelled water method. Second, to compare the SDQ's performance in estimating energy, nutrient and food intakes with a sixty-six-item FFQ used in large-scale Swedish epidemiological research.

Design: Cross-sectional.

Setting: Umeå, Sweden.

Subjects: In total, sixty-five non-pregnant women, of whom thirty-one were overweight or obese, and twenty-five pregnant, normal-weight women completed the protocol.

Results: On average, the SDQ captured $78 \%$ and $79 \%$ of absolute TEE in the non-pregnant and pregnant normal-weight women, respectively. Furthermore, the SDQ captured an average of $57 \%$ of TEE in the overweight/obese nonpregnant women. The Spearman correlation of EI and TEE was significant in the overweight and obese women only $(\rho=0 \cdot 37,95 \%$ CI $0 \cdot 02,0 \cdot 64)$. There was no significant difference between the SDQ and the more extensive FFQ in the ability to assess EI when compared with TEE. Intakes of most nutrients and foods were significantly higher when assessed with the SDQ compared with the FFQ. Conclusions: A new short dietary questionnaire with an alternative design underestimated EI of non-pregnant and pregnant, overweight and obese women on a group level but was able to rank the overweight/obese women according to EI. Furthermore, the short questionnaire captured as much or more of the energy, nutrient and food intakes of non-pregnant normal-weight and overweight/obese women on the group level as a traditional, more extensive FFQ.
\end{abstract}

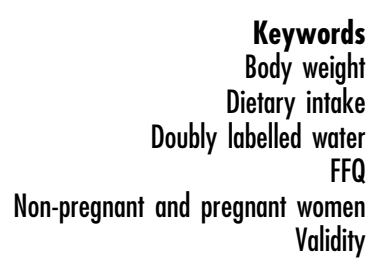

There is a need for quick and easy-to-administer dietary assessment tools with low respondent burden that give valid intake data in large-scale population studies and that are able to rank participants according to intake. The FFQ method is most often used in epidemiological studies ${ }^{(1)}$, since it is easy to administer at a relatively low cost and estimates habitual dietary intake over an extended time period $^{(2)}$. A valid FFQ can be used to rank individuals according to reported intake and, if it includes portion size estimations, to assess absolute individual intakes ${ }^{(3)}$.

$\uparrow$ Correspondence address: Department of Food and Nutrition, and Sport Science, Box 300, SE-405 30 Gothenburg, Sweden.
However, a problem with all self-report dietary assessment methods is the misreporting of energy intake (EI) ${ }^{(4)}$. Dietary intake in young, weight-conscious women can be especially difficult to assess accurately, and previous studies in women show underestimation of EI when assessed using FFQ by comparison with total energy expenditure (TEE) measured by the doubly labelled water (DLW) method ${ }^{(5,6)}$. Furthermore, it has repeatedly been shown that for overweight and obese individuals EI is underestimated to a higher extent than for their normalweight counterparts ${ }^{(6-9)}$. One biomarker study suggested that the FFQ worked better in detecting the higher EI in obese women when compared with the $24 \mathrm{~h} \mathrm{recall}^{(10)}$. 
In a review of 227 different FFQ the number of items queried ranged from five to 350 , with a mean of eighty-eight items ${ }^{(11)}$. The review included FFQ aimed at assessing the whole diet or parts of the diet, e.g. intake of fruits and vegetables. On average, study participants take 15-30 min to complete a typical FFQ ${ }^{(12)}$, suggesting a need for shorter and less burdensome dietary questionnaires for use in large epidemiological studies. Shorter versions of established FFQ, retaining the same design, have been developed and validated, e.g. a short version of the Block questionnaire, which showed lower absolute values and correlation coefficients than the original FFQ for macronutrients when compared with food records ${ }^{(13)}$. In a meta-analysis, correlation coefficients for most nutrients were higher for FFQ of 200 items than for FFQ of 100 items when compared with reference methods ${ }^{(14)}$. However, although more extensive FFQ generally perform better, this may increase the risk of non-compliance. Most FFQ query the frequency of consumption with given response alternatives (e.g. 'never' to ' 4 times per day') and use standard portion sizes for calculation of energy and nutrient intakes. Questionnaires with an alternative design are less common ${ }^{(15)}$. Integrating the amounts of food consumed with the frequencies of consumption in the FFQ could possibly make it easier for the participants to validly report their intakes and improve the estimation of consumed amounts.

There has been limited research validating alternative methods in younger female populations who are known to be prone to under-reporting. Moreover, study subgroups of overweight and pregnant women are seldom recruited specifically, in addition to normal-weight, nonpregnant subjects.

Therefore, the primary aim of the present study was to evaluate the ability of a short dietary questionnaire (SDQ) to assess EI in normal-weight/overweight/obese non-pregnant women, on group and individual levels compared with objectively measured TEE. A secondary aim was to compare the SDQ's performance in estimating energy, nutrient and food intakes with a sixty-six-item FFQ used in large-scale Swedish epidemiological research among normal-weight/overweight/obese non-pregnant women.

\section{Materials and methods}

\section{Participants and setting}

In previous work we have shown that a sample size of thirty to fifty subjects is sufficient to allow the statistical comparisons between Actiheart (or uniaxial accelerometry) and DLW estimates of energy expenditure ${ }^{(16,17)}$. Recognizing that nutritional questionnaires are more error prone than objective physical activity measures, because we were interested in testing additional hypotheses and because our study design and setting differed from the earlier studies $^{(16,17)}$, we sought to recruit $>100$ women for the current study.

In 2008-2009, seventy-three non-pregnant normalweight or overweight/obese women born in 1973-1988 and living in the county of Västerbotten, northern Sweden, were recruited through advertisements in local media and by word of mouth. Normal-weight, overweight and obese women were defined as having a BMI corresponding to $<25 \cdot 0,25 \cdot 0-29 \cdot 9$ and $\geq 30 \cdot 0 \mathrm{~kg} / \mathrm{m}^{2}$, respectively $^{(18)}$. In addition, thirty-five pregnant women with similar demographic characteristics (i.e. age, region of residence, income level and education level) were recruited at 8-16 weeks of gestation through local antenatal clinics with the help of midwives. The pregnant women participated in the study at 28-32 weeks of gestation. Exclusion criteria were recent cardiovascular events, recent physically debilitating surgical procedures, unmanaged serious psychiatric disorders, dependency on illicit drugs and an inability to commit fully to the study protocol. The study was conducted according to the guidelines laid down in the Declaration of Helsinki and all procedures involving human subjects were approved by the Regional Ethical Review Board in Umeå, Sweden. Written informed consent was obtained from all participants.

An SDQ was completed unsupervised by the participants during a visit to the Clinical Research Center, Umeå University hospital, and a sixty-six-item FFQ was completed at home after a $10 \mathrm{~d}$ DLW method measurement period. Both questionnaires assess dietary intake as well as intake of dietary supplements; however, the dietary supplement data were not included in the present study since these questions were not comparable between the SDQ and the FFQ. Since the FFQ refers to the period of the previous 12 months it was not included in the analysis of the pregnant women whose diet can be assumed to have been unstable during this period. The SDQ, however, refers to the period of the previous 3 months, thereby reflecting the intake during pregnancy, and was therefore used in the analyses of the pregnant women.

\section{The short dietary questionnaire}

The SDQ was developed with the aim to be short, fit on one A4-page and take less than $10 \mathrm{~min}$ to complete, but still capture the majority of an individual's dietary intake and make it possible to calculate energy and nutrient intakes. The SDQ was developed to be easier and quicker for all participants to complete regardless of weight status or pregnancy. The questionnaire was tested in a pilot study of women with nutrition as professional background and thereafter was revised (C Larsson, unpublished results) before it was used in the present study. The reason for choosing women with nutrition as profession to participate in the pilot study was to obtain relevant and qualified feedback on the design and questions of the SDQ. The design of the SDQ (see online supplementary material) 
differs from that of a traditional FFQ and consists of questions about frequency and portion size/amount of intake of thirty-nine foods/food groups/dishes, including beverages, as well as dietary supplements during the previous 3 months. The thirty-nine foods/food groups/ dishes are representative of a mixed Swedish diet and contribute significantly to intakes of energy, Ca, $\mathrm{Fe}$, Se and $\mathrm{Zn}$ according to a Swedish national diet survey ${ }^{(19)}$. These micronutrients were selected to guide the development of the SDQ since it was shown in the national diet survey that Swedish women do not reach the recommended intakes of $\mathrm{Fe}$ and $\mathrm{Se}$, and intake of $\mathrm{Ca}$ is especially important in younger ages when bone formation peaks. The selected foods were also shown to contribute to a majority of the $\mathrm{Zn}$ intake. Standard weight and portion sizes of food/food groups/dishes were estimated through weighing of several food items and portions of dishes as well as by using standard portions from weight tables developed by the National Food Agency ${ }^{(20)}$. Energy and nutrient contents of the thirty-nine foods/food groups/dishes were obtained by aggregating and calculating mean contents of representative foods from the Swedish Food Database (version 2009-05-19); e.g. the energy and nutrient contents of 'white bread' in the SDQ were obtained by calculation of the mean energy and nutrient contents of five different types of white bread from the food database. Average values of the thirty-nine foods were entered as new foods in the nutrition calculation software Dietist XP version 3·1 (Kost och Näringsdata $A B$, Bromma, Sweden), which uses the Swedish Food Database (version 2009-05-19), and used for calculation of assessed energy and nutrient intake of the SDQ.

\section{FFQ}

The FFQ has previously been validated in the same general population to which participants in the present study belong ${ }^{(21,22)}$. The FFQ initially included eighty-four food items, but was later shortened by merging or removing foods. Therefore, the FFQ used in the present study consists of sixty-six listed foods/food groups/ dishes, including beverages, and queries dietary intake during the previous year. The nine frequencies of consumption are 'never', 'a few times per year', ' $1-3$ times per month', 'once a week', '2-3 times a week', ' $4-6$ times a week', 'once a day', ' $2-3$ times a day' and ' 4 or more times per day'. Reported frequencies were converted to the number of intakes per day and multiplied by a portion size value. The assessment of portion size is reported separately for (i) potatoes, rice and pasta, (ii) meat and fish and (iii) vegetables, and is aided by four colour photographs showing plates with increasing amounts. Other portion size values were natural portion sizes (e.g. an apple) or age- and sex-specific averages obtained from a national survey ${ }^{(20,23)}$. Energy and nutrient intakes were calculated by using the nutrition calculation software Stor
MATs (Rudans Lättdata, Västerås, Sweden) which uses the Swedish Food Database version 1994 (for macronutrients) and 2009 (for micronutrients).

\section{Doubly labelled water method}

The DLW method was used to determine TEE. Participants were invited to the Clinical Research Center at Umeå University hospital in the morning following an $8 \mathrm{~h}$ overnight fast. Their weight was measured to the nearest $0 \cdot 1 \mathrm{~kg}$ using a calibrated digital scale, height to the nearest $0.5 \mathrm{~cm}$ using a wall-mounted stadiometer and BMI $\left(\mathrm{kg} / \mathrm{m}^{2}\right)$ was calculated. Subsequently, a pre-dose urine sample was collected following which the participants were given an individually prepared oral dose of stableisotope-enriched water $\left(0.07 \mathrm{~g}^{2} \mathrm{H}_{2} \mathrm{O}\right.$ and $0.174 \mathrm{~g} \mathrm{H}_{2}{ }^{18} \mathrm{O}$ per $\mathrm{kg}$ body weight). Further urine samples were collected for each of the following $10 \mathrm{~d}$, of which the samples from days $1-3$ and $8-10$ were analysed. The participant noted the time for each sample in a log. Urine samples were kept in plastic vials at $+4-8^{\circ} \mathrm{C}$ until returned and then frozen at $-20^{\circ} \mathrm{C}$ pending analysis. Isotopic enrichments of dose and urine samples were analysed at MRC Human Nutrition Research, Cambridge, UK, using methods described in detail elsewhere ${ }^{(24)}$.

\section{Exclusions}

All 108 recruited women filled out the SDQ, 106 filled out the FFQ and 102 successfully underwent DLW measurements. The SDQ was developed to assess a traditional mixed Swedish diet; thus, six women who were vegetarians or did not eat red meat were excluded from the analyses, together with two women who did not fully complete the SDQ. Ten women not fully completing the FFQ were excluded from the analyses involving the FFQ. Furthermore, three of the pregnant women were obese. Considering that this group was too small to be analysed separately, they were excluded from the analysis. Some of the women had missing data with more than one of the methods. In total, ninety women were included in analyses: sixty-one non-pregnant women with different weight status who had a complete set of data from SDQ, FFQ and DLW measurements; an additional four nonpregnant women who had complete SDQ and DLW measurements but lacked complete FFQ; and twenty-five normal-weight pregnant women who had complete SDQ and DLW measurements.

\section{Statistical analysis}

Statistical analyses were performed in IBM SPSS Statistics version 20 and $P$ values of $\leq 0.05$ (two-sided) were considered significant. The variables were checked for normality of distribution by using the Shapiro-Wilk test. All foods and several nutrients and participant characteristics were non-normally distributed and non-parametric statistics were therefore principally used. Data are presented as medians, interquartile ranges, percentage proportions, 
Spearman correlation coefficients, means and standard deviations.

Differences in characteristics between normal-weight non-pregnant women and overweight/obese non-pregnant women and normal-weight pregnant women, respectively, were analysed using the Mann-Whitney $U$ test.

Validity of assessed EI was analysed using three different approaches:

1. Bland-Altman analyses of the agreement of EI assessed with the SDQ (for the non-pregnant and pregnant women) and the FFQ (only for the nonpregnant women) with TEE obtained from DLW.

2. One-sample Wilcoxon signed-rank tests were used to analyse accuracy of assessment on the group level $\left(\mathrm{EI}_{\mathrm{SDQ}}\right.$ - TEE and $\mathrm{EI}_{\mathrm{FFQ}}$ - TEE, respectively, for the non-pregnant women, and $\mathrm{EI}_{\mathrm{SDQ}}$ - TEE for the pregnant women). In the non-pregnant women, differences in accuracy between normal-weight and overweight/obese women were analysed using the Mann-Whitney $U$ test.

3. Spearman correlation coefficients were calculated between $\mathrm{EI}_{\mathrm{SDQ}}$ and TEE for the non-pregnant and pregnant women and between $\mathrm{EI}_{\mathrm{FFQ}}$ and TEE for the non-pregnant women. For the non-pregnant women, results for normal-weight and overweight/obese women were calculated separately.

In the non-pregnant women, within-subject difference in accuracy of assessed EI between the SDQ and the FFQ was analysed using the Wilcoxon signed-rank test. The Spearman correlation coefficient was calculated between $\mathrm{EI}_{\mathrm{SDQ}}-\mathrm{TEE}$ and $\mathrm{EI}_{\mathrm{FFQ}}-\mathrm{TEE}$ in order to investigate the association of errors of the two questionnaires.

Differences between the SDQ and FFQ in the ability to assess foods and nutrients in the non-pregnant women were analysed using the Wilcoxon signed-rank test. Spearman correlation coefficients were also calculated.

\section{Results}

\section{Participant characteristics}

Characteristics, EI and TEE of the participants are shown in Table 1 . The pregnant women were normal weight at the time of recruitment (8-16 weeks of gestation; data not shown).

\section{Ability of the short dietary questionnaire to assess energy intake}

The agreement between $\mathrm{EI}_{\mathrm{SDQ}}$ and TEE in the nonpregnant women is displayed in a Bland-Altman plot in Fig. 1(a). The SDQ underestimated EI by $30 \%$ on the group level $(P<0 \cdot 001)$, corresponding to -3379 (median) and -3434 (mean) $\mathrm{kJ} / \mathrm{d}$ (Table 1, Fig. 1). Three women were outside the limits of agreement $(-8382$, $1514 \mathrm{~kJ} / \mathrm{d}$ ) and three women had a reported EI within $\pm 5 \%$ of their individually measured TEE. Reported EI of four women was $>5 \%$ and of fifty-eight women was $<5 \%$ of individually measured TEE. The underestimation of EI was higher in those with higher energy expenditure.

As shown in Table 1, the SDQ underestimated EI of overweight/obese women to a greater extent than EI of normal-weight women, 43\% compared with $22 \%$ $(P=0 \cdot 02)$. However, the correlation between $\mathrm{EI}_{\mathrm{SDQ}}$ and TEE was statistically significant $(P=0.04)$ in the overweight/obese women but not in the normal-weight women.

Figure 2 illustrates the agreement between $\mathrm{EI}_{\mathrm{SDQ}}$ and TEE in the pregnant women. In comparison with TEE, $\mathrm{EI}_{\mathrm{SDQ}}$ was underestimated by $21 \%(P=0 \cdot 002)$, corresponding to -2224 (median) and -2415 (mean) $\mathrm{kJ} / \mathrm{d}$. Two women were outside the limits of agreement $(-4044$, $8873 \mathrm{~kJ} / \mathrm{d}$ ) and one woman had a reported EI within $\pm 5 \%$ of her individually measured TEE. Reported EI of three women was $>5 \%$ and of twenty-one women was $<5 \%$ of individually measured TEE. The underestimation of EI was higher in those with higher energy expenditure. The correlation between $\mathrm{EI}_{\mathrm{SDQ}}$ and TEE was not statistically significant $(P=0 \cdot 97$; Table 1$)$.

\section{Ability of the short dietary questionnaire to assess intakes in comparison with the sixty-six-item FFQ}

Comparison of agreement between EI assessed with SDQ and FFQ, respectively, and TEE is displayed in Bland-Altman plots in Figs 1(a) and 1(b). EI assessed with the SDQ was $70 \%$ of the TEE, while EI assessed with the FFQ was $66 \%$ of the TEE (Table 1). However, the difference in underestimation between the two questionnaires ( $n$ 61) was not statistically significant $(P=0 \cdot 21)$. The Spearman correlation coefficient between $\mathrm{EI}_{\mathrm{SDQ}}$ - TEE and $\mathrm{EI}_{\mathrm{FFQ}}$ - TEE was 0.62 $(P<0.001)$, indicating a correlated error structure.

Intakes of five out of eight nutrients (protein, carbohydrate, $\mathrm{Ca}$, Se and $\mathrm{Zn}$ ) adjusted for EI (amount per MJ) were significantly higher when assessed with the SDQ than with the FFQ, while the intakes of fat and Fe were significantly lower $(P<0 \cdot 001$; Table 2$)$. This was true also when the analyses were performed with unadjusted intakes (data not shown). Spearman correlations for the eight nutrients assessed with the SDQ and the FFQ ranged from 0.37 to $0.66(P<0 \cdot 01)$.

In total, twenty-six of the foods/food groups included in the SDQ were also found in the FFQ (Table 3). Intakes of fifteen out of these were significantly higher for the SDQ compared with the FFQ and for nine of the foods/food groups there were no statistically significant differences in intakes. For two foods/food groups ('Milk/ sour milk/yoghurt 3\% fat' and 'Chips and other snacks') the FFQ assessed significantly higher amounts than the SDQ. Spearman correlations for foods assessed with the SDQ and the FFQ ranged from $0 \cdot 47$ to $0 \cdot 81(P<0 \cdot 001)$, 
Table 1 Participant characteristics, energy intake (EI) assessed with questionnaires, measured total energy expenditure from doubly labelled water (TEE $E_{D L W}$ ), difference EI - TEE $E_{D L W}$, ratio El:TEE $E_{D L W}$ and Spearman correlation coefficients for El and TEE $E_{D L W}$ of non-pregnant and pregnant women by weight status, Västerbotten, northern Sweden, 2008-2009

\begin{tabular}{|c|c|c|c|c|c|c|c|c|c|c|}
\hline & \multicolumn{7}{|c|}{ Non-pregnant } & \multirow{2}{*}{\multicolumn{2}{|c|}{$\begin{array}{r}\text { Pregnant } \\
\text { Normal weight* }^{*}(n 25)\end{array}$}} & \multirow[b]{3}{*}{$P$ value } \\
\hline & \multicolumn{2}{|c|}{ All $(n 65)$} & \multicolumn{2}{|c|}{ Normal weight* (n 34) } & \multicolumn{2}{|c|}{ Overweight/obese* $(n$ 31) } & \multirow[b]{2}{*}{$P$ valuet } & & & \\
\hline & $\begin{array}{l}\text { Median or } n \\
\quad \text { or } \rho\end{array}$ & $\begin{array}{c}\text { IQR or } \% \text { or } \\
95 \% \mathrm{Cl}\end{array}$ & $\begin{array}{l}\text { Median or } n \\
\quad \text { or } \rho\end{array}$ & $\begin{array}{c}\text { IQR or \% or } \\
95 \% \mathrm{Cl}\end{array}$ & $\begin{array}{l}\text { Median or } n \\
\quad \text { or } \rho\end{array}$ & $\begin{array}{l}\text { IQR or } \% \text { or } \\
95 \% \mathrm{Cl}\end{array}$ & & $\begin{array}{l}\text { Median or } n \\
\quad \text { or } \rho\end{array}$ & $\begin{array}{l}\text { IQR or \% or } \\
95 \% \mathrm{Cl}\end{array}$ & \\
\hline \multicolumn{11}{|c|}{ Group characteristics, median and IQR } \\
\hline Age (years) & $29 \cdot 2$ & $6 \cdot 6$ & $28 \cdot 7$ & $5 \cdot 4$ & $30 \cdot 0$ & 8.9 & $0 \cdot 70$ & $31 \cdot 5$ & $3 \cdot 8$ & 0.03 \\
\hline Weight (kg) & $74 \cdot 2$ & $28 \cdot 8$ & $60 \cdot 1$ & $10 \cdot 9$ & $88 \cdot 4$ & $18 \cdot 4$ & $<0.001$ & $70 \cdot 3$ & $8 \cdot 1$ & $<0.001$ \\
\hline Height (cm) & $168 \cdot 0$ & $8 \cdot 8$ & $167 \cdot 8$ & $12 \cdot 4$ & $168 \cdot 5$ & $8 \cdot 5$ & $0 \cdot 83$ & $165 \cdot 5$ & $9 \cdot 8$ & 0.71 \\
\hline BMI $\left(\mathrm{kg} / \mathrm{m}^{2}\right)$ & $24 \cdot 7$ & $8 \cdot 8$ & $22 \cdot 3$ & $2 \cdot 6$ & $31 \cdot 0$ & $5 \cdot 8$ & $<0.001$ & $25 \cdot 2$ & $3 \cdot 6$ & $<0.001$ \\
\hline \multicolumn{11}{|l|}{ Educational level, $n$ and $\%$} \\
\hline Vocational training & 1 & $1 \cdot 5$ & 1 & $2 \cdot 9$ & 0 & 0 & $\mathrm{~N} / \mathrm{A}$ & 1 & $4 \cdot 0$ & N/A \\
\hline Upper secondary school & 26 & $40 \cdot 1$ & 9 & $26 \cdot 5$ & 17 & $54 \cdot 8$ & $\mathrm{~N} / \mathrm{A}$ & 4 & $16 \cdot 0$ & $\mathrm{~N} / \mathrm{A}$ \\
\hline University/college & 37 & $56 \cdot 9$ & 23 & $67 \cdot 7$ & 14 & $45 \cdot 2$ & $\mathrm{~N} / \mathrm{A}$ & 19 & $76 \cdot 0$ & N/A \\
\hline Missing data & 1 & $1 \cdot 5$ & 1 & $2 \cdot 9$ & 0 & 0 & $\mathrm{~N} / \mathrm{A}$ & 1 & $4 \cdot 0$ & $\mathrm{~N} / \mathrm{A}$ \\
\hline \multicolumn{11}{|c|}{ Energy variables, median and IQR } \\
\hline $\mathrm{TEE}_{\mathrm{DLW}}(\mathrm{kJ} / \mathrm{d})$ & 10647 & 2042 & 10301 & 1266 & 11969 & 1899 & $<0.001$ & 11133 & 1599 & 0.02 \\
\hline $\mathrm{El}_{\mathrm{SDQ}}(\mathrm{kJ} / \mathrm{d})$ & 7553 & 3315 & 8118 & 2839 & 6788 & 3770 & $0 \cdot 37$ & 8502 & 4368 & 0.35 \\
\hline $\operatorname{El}_{\mathrm{FFQ}}(\mathrm{kJ} / \mathrm{d}) \S$ & 6872 & 2425 & 7121 & 2075 & 6686 & 2378 & 0.06 & $\mathrm{~N} / \mathrm{A}$ & $\mathrm{N} / \mathrm{A}$ & $\mathrm{N} / \mathrm{A}$ \\
\hline $\mathrm{El}_{\mathrm{SDQ}}-\mathrm{TEE} \mathrm{E}_{\mathrm{DLW}}(\mathrm{kJ} / \mathrm{d})$ & -3379 & 3894 & -2304 & 3133 & -4800 & 3573 & 0.01 & -2224 & 4063 & 0.91 \\
\hline $\mathrm{EI}_{\mathrm{FFQ}}-\mathrm{TEE}_{\mathrm{DLW}}(\mathrm{kJ} / \mathrm{d}) \S$ & -4002 & 3423 & -2840 & 2227 & -5189 & 3520 & $<0.001$ & $\mathrm{~N} / \mathrm{A}$ & $\mathrm{N} / \mathrm{A}$ & N/A \\
\hline $\mathrm{El}_{\mathrm{SDQ}}: \mathrm{TEE}_{\mathrm{DLW}}$ & $0 \cdot 70$ & 0.33 & $0 \cdot 78$ & $0 \cdot 31$ & 0.57 & $0 \cdot 33$ & 0.02 & 0.79 & 0.39 & 0.83 \\
\hline $\mathrm{El}_{\mathrm{FFQ}}: \mathrm{TEE}_{\mathrm{DLW}} \S$ & 0.66 & $0 \cdot 22$ & $0 \cdot 71$ & $0 \cdot 21$ & 0.54 & $0 \cdot 26$ & 0.001 & N/A & N/A & N/A \\
\hline \multicolumn{11}{|l|}{ Correlations, $\rho$ and $95 \% \mathrm{Cl}$} \\
\hline $\mathrm{El}_{\mathrm{SDQ}}$ and $\mathrm{TEE} \mathrm{E}_{\mathrm{DLW}}$ & $0 \cdot 14$ & $-0 \cdot 11,0 \cdot 37$ & $0 \cdot 13$ & $-0.21,0.45$ & $0 \cdot 37$ & $0.02,0.64$ & $\mathrm{~N} / \mathrm{A}$ & -0.007 & $-0.40,0.39$ & $N / A$ \\
\hline $\mathrm{El}_{\mathrm{FFQ}}$ and $\mathrm{TE} \mathrm{E}_{\mathrm{DLw}} \mathrm{s}$ & -0.05 & $-0 \cdot 30,0 \cdot 20$ & 0.08 & $-0.28,0.42$ & $0 \cdot 17$ & $-0.20,0.50$ & $\mathrm{~N} / \mathrm{A}$ & N/A & $\mathrm{N} / \mathrm{A}$ & $\mathrm{N} / \mathrm{A}$ \\
\hline $\mathrm{El}_{\mathrm{SDQ}}$ and $\mathrm{El}_{\mathrm{FFQ}} \S$ & 0.48 & $0.25,0.65$ & 0.49 & $0 \cdot 17,0 \cdot 72$ & 0.43 & $0.08,0.68$ & $\mathrm{~N} / \mathrm{A}$ & $\mathrm{N} / \mathrm{A}$ & N/A & $\mathrm{N} / \mathrm{A}$ \\
\hline
\end{tabular}

$\rho$, Spearman correlation; IQR, interquartile range; SDQ, short dietary questionnaire; FFQ, Swedish sixty-six-item FFQ; N/A, not applicable DAccording to cut-off values defined by the $\mathrm{WHO}^{(18)}$.

Differences in characteristics between normal-weight and overweight/obese participants analysed using the Mann-Whitney $U$ test.

fDifferences in characteristics between non-pregnant and pregnant normal-weight participants analysed using the Mann-Whitney $U$ test.

Sixty-one participants with available FFQ data. 

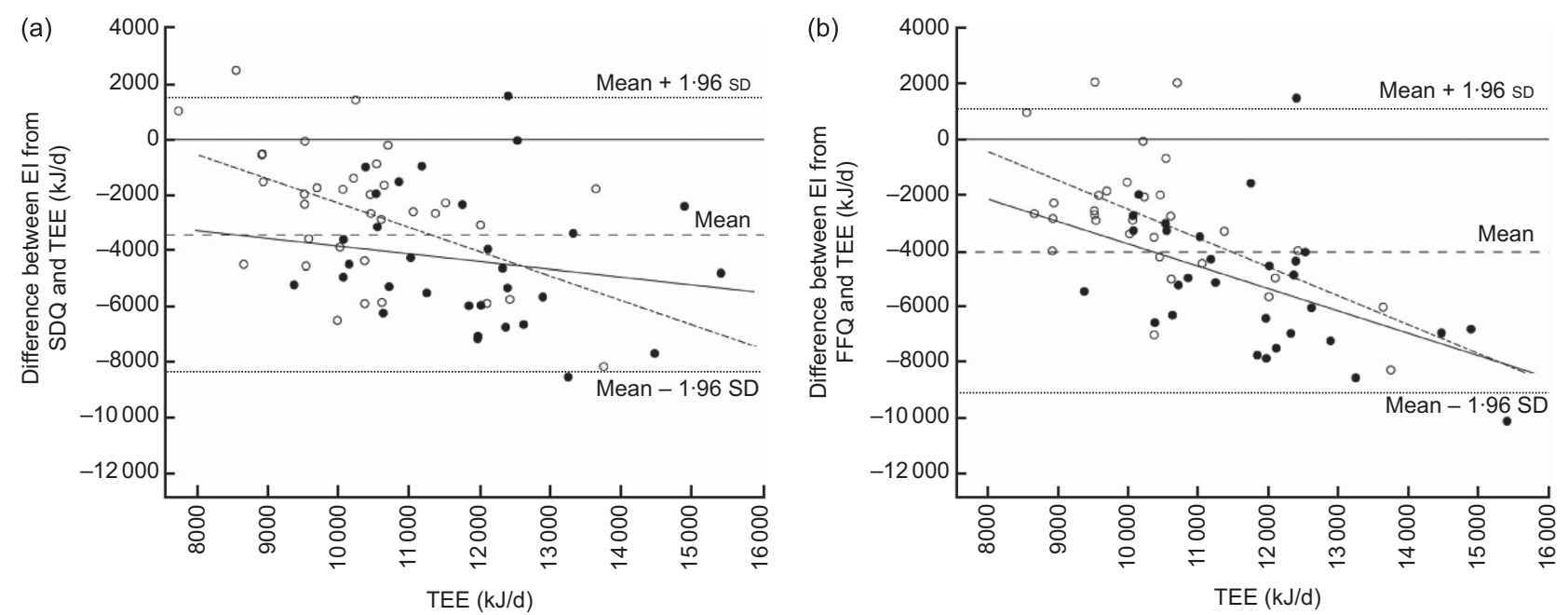

Fig. 1 Bland-Altman plots comparing energy intake (EI) of non-pregnant women ( $n$ 65) assessed with (a) a short dietary questionnaire (SDQ) and (b) a more extensive FFQ ( $n$ 61) against total energy expenditure (TEE) measured with the doubly labelled water method, Västerbotten, northern Sweden, 2008-2009. Normal-weight women are displayed as $\bigcirc$ and overweight/ obese women as $\bullet$. Difference between EI and TEE is shown on the $y$-axis and TEE on the $x$-axis. The mean difference is displayed as ----- and the limits of agreement (mean $\pm 1.96 \mathrm{sD}$ ) are displayed as $\cdots \cdots$. The regression lines are displayed for normal-weight women $(----)$ ) and overweight/obese women $(-)$

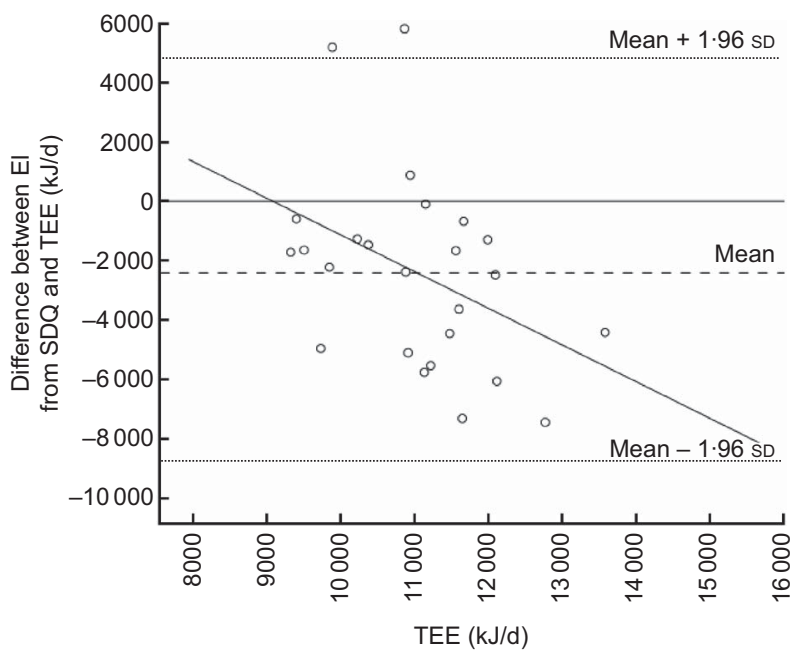

Fig. 2 Bland-Altman plot comparing energy intake (EI) of normal-weight pregnant women $(n 25)$ assessed with a short dietary questionnaire (SDQ) to total energy expenditure (TEE) measured with the doubly labelled water method, Västerbotten, northern Sweden, 2008-2009. Difference between EI and TEE is displayed on the $y$-axis and TEE on the $x$-axis. The mean difference is displayed as - - - - - and the limits of agreement (mean $\pm 1.96 \mathrm{sD}$ ) are displayed as.....

except high-fat milk/sour milk/yoghurt for which the correlation was $0 \cdot 28(P=0 \cdot 03)$.

\section{Discussion}

The SDQ underestimated the EI of non-pregnant and pregnant, normal-weight and overweight/obese women compared with objectively measured TEE, but it was able to rank the overweight/obese women reasonably well according to EI. The FFQ underestimated EI to a similar extent as the SDQ and was not able to rank the participants' EI. Intakes of certain macronutrients and minerals, as well as intakes of most foods, were significantly higher when assessed with the SDQ compared with the FFQ. However, since no criterion measure for these variables was used in the present study, it is not possible to determine which instrument is more accurate for assessing foods and nutrients. The results suggest that the shorter SDQ is as accurate as a traditionally designed and more extensive FFQ in estimating EI, nutrients and foods.

There has been some discussion about whether women generally under-report more than men, but evidence points to no consistent differences in reporting accuracy between the sexes ${ }^{(4,25,26)}$. However, a number of previous validation studies of FFQ in women have reported underestimation of EI when compared with TEE measured by the DLW method ${ }^{(5,6,26,27)}$. In one study, EI assessed using a 180-item FFQ was underestimated by $10 \%$ among Norwegian women ${ }^{(5)}$, and in a large study, conducted in the USA, the underestimation of EI was $34 \%$ using a 124-item $\mathrm{FFQ}^{(26)}$. The results of the present study showed that the women who were overweight or obese underestimated their EI to a greater extent than the normal-weight women using both the SDQ and the FFQ, which is consistent with previous results of studies relating misreporting of EI to body weight ${ }^{(28,29)}$. The trend of higher underestimation of EI with higher energy needs seen in both normal-weight and overweight/obese women in the present study has also been shown previously $^{(27)}$. In line with our recruitment goals, almost 
Table 2 Intake of nutrients assessed with a short dietary questionnaire (SDQ) and a more extensive FFQ in sixty-one normal-weight/ overweight/obese non-pregnant women, Västerbotten, northern Sweden, 2008-2009

\begin{tabular}{|c|c|c|c|c|c|}
\hline & \multicolumn{2}{|c|}{ SDQ } & \multicolumn{2}{|c|}{ FFQ } & \multirow[b]{2}{*}{$P$ value* } \\
\hline & Median & IQR & Median & IQR & \\
\hline \multicolumn{6}{|c|}{ Nutrients for which the SDQ gave a higher estimated intake compared with the FFQ } \\
\hline Protein $(\mathrm{g} / \mathrm{MJ}$ per $\mathrm{d})$ & $10 \cdot 4$ & $2 \cdot 0$ & $9 \cdot 0$ & $1 \cdot 9$ & $<0.001$ \\
\hline Carbohydrate (g/MJ per d) & $28 \cdot 8$ & $4 \cdot 1$ & $26 \cdot 9$ & $4 \cdot 9$ & $<0.001$ \\
\hline $\mathrm{Ca}(\mathrm{mg} / \mathrm{MJ}$ per d) & $105 \cdot 9$ & $45 \cdot 9$ & $82 \cdot 9$ & $41 \cdot 3$ & $<0.001$ \\
\hline Se $(\mu \mathrm{g} / \mathrm{MJ}$ per $\mathrm{d})$ & $4 \cdot 6$ & $2 \cdot 1$ & $3 \cdot 6$ & $1 \cdot 4$ & $<0.001$ \\
\hline $\mathrm{Zn}(\mathrm{mg} / \mathrm{MJ}$ per d) & $1 \cdot 4$ & 0.3 & $1 \cdot 1$ & $0 \cdot 3$ & $<0.001$ \\
\hline \multicolumn{6}{|c|}{ Nutrients for which the FFQ gave a higher estimated intake compared with the SDQ } \\
\hline Fat (g/MJ per d) & $7 \cdot 9$ & $1 \cdot 7$ & $10 \cdot 2$ & $2 \cdot 2$ & $<0.001$ \\
\hline $\mathrm{Fe}(\mathrm{mg} / \mathrm{MJ}$ per $\mathrm{d})$ & $1 \cdot 2$ & $0 \cdot 3$ & $1 \cdot 6$ & $0 \cdot 3$ & $<0.001$ \\
\hline \multicolumn{6}{|c|}{ Nutrient for which no statistically significant difference in intake between the SDQ and the FFQ was found } \\
\hline Alcohol (g/MJ per d)t & 0.3 & 0.5 & 0.4 & 0.4 & 0.73 \\
\hline
\end{tabular}

$\mathrm{IQR}$, interquartile range.

Data are presented as median and interquartile range of energy-adjusted intakes.

${ }^{\star} P$ value derived from the Wilcoxon signed-rank test for the difference in intakes assessed with the two questionnaires.

tFor the fifty-eight women who reported to consume alcohol.

Table 3 Intake of foods/food groups assessed with a short dietary questionnaire (SDQ) and a more extensive FFQ in sixty-one normalweight/overweight/obese non-pregnant women, Västerbotten, northern Sweden, 2008-2009

\begin{tabular}{|c|c|c|c|c|c|}
\hline & \multicolumn{2}{|c|}{$\operatorname{SDQ}(g / d)$} & \multicolumn{2}{|c|}{$\mathrm{FFQ}(\mathrm{g} / \mathrm{d})$} & \multirow[b]{2}{*}{$P$ value* } \\
\hline & Median & IQR & Median & IQR & \\
\hline \multicolumn{6}{|c|}{ Foods/food groups for which the SDQ gave a higher estimated intake compared with the FFQ } \\
\hline Bread & 90 & 90 & 47 & 43 & $<0.001$ \\
\hline Butter/margarine on bread, $40 \%$ fat & 0 & 7 & 0 & 3 & 0.003 \\
\hline Cheese, $17 \%$ fat & 0 & 10 & 2 & 7 & 0.01 \\
\hline Cheese, $28 \%$ fat & 10 & 29 & 6 & 12 & $<0.001$ \\
\hline Milk/sour milk/yoghurt, $1-1.5 \%$ fat & 60 & 200 & 22 & 112 & 0.002 \\
\hline Fruits and berries & 206 & 138 & 127 & 138 & $<0.001$ \\
\hline Rice & 47 & 47 & 25 & 31 & 0.003 \\
\hline Pasta & 103 & 103 & 72 & 43 & $<0.001$ \\
\hline Fish & 24 & 32 & 21 & 19 & 0.004 \\
\hline Poultry & 36 & 42 & 21 & 39 & 0.008 \\
\hline Cream/crème fraîche & 14 & 22 & 5 & 10 & $<0.001$ \\
\hline Biscuits/cookies/buns/cake & 12 & 16 & 9 & 12 & 0.04 \\
\hline Chocolate and sweets & 14 & 22 & 7 & 13 & 0.004 \\
\hline Juice/syrup/soft drinks & 100 & 143 & 32 & 72 & $<0.001$ \\
\hline Spiritst & 0 & 7 & 0 & 4 & $<0.001$ \\
\hline \multicolumn{6}{|c|}{ Foods/food groups for which the FFQ gave a higher estimated intake compared with the SDQ } \\
\hline Milk/sour milk/yoghurt, $3 \%$ fat & 0 & 21 & 33 & 121 & $<0.001$ \\
\hline Chips and other snacks & 1 & 2 & 2 & 2 & $<0.001$ \\
\hline \multicolumn{6}{|c|}{ Foods/food groups for which no statistically significant difference in intake between the SDQ and the FFQ was found } \\
\hline Butter/margarine on bread, $60-80 \%$ fat & 2 & 6 & 3 & 9 & $0 \cdot 13$ \\
\hline Milk/sour milk/yoghurt, $0.5 \%$ fat & 30 & 150 & 33 & 207 & $0 \cdot 36$ \\
\hline Vegetables & 71 & 76 & 90 & 114 & 0.08 \\
\hline Boiled potatoes & 50 & 44 & 58 & 64 & 0.23 \\
\hline Fried potatoes/pommes frites & 0 & 20 & 11 & 12 & 0.39 \\
\hline Meat & 70 & 75 & 78 & 52 & 0.47 \\
\hline Ice cream & 5 & 13 & 6 & 6 & 0.55 \\
\hline Beert & 7 & 25 & 2 & 29 & 0.73 \\
\hline Winet & 14 & 27 & 18 & 17 & 0.95 \\
\hline
\end{tabular}

IQR, interquartile range.

Data are presented as median and interquartile range.

${ }^{*} P$ value derived from the Wilcoxon signed-rank test for the difference in intakes assessed with the two questionnaires.

tFor the fifty-eight women who reported to consume alcohol.

half of the non-pregnant participants were overweight or obese in the present study, and therefore presumably more prone to under-reporting than had the sample been representative of the general population.

In a previous study, the evaluated FFQ was able to rank women according to EI when compared with TEE measured using the DLW method ${ }^{(27)}$. In another study, which also used TEE measured using the DLW method for comparison, the evaluated FFQ was not able to rank women according to $\mathrm{EI}^{(5)}$. In the present study, EI assessed with the SDQ correlated significantly with TEE, but only in the overweight/obese women. The FFQ was 
not able to rank the participants according to EI in any of the groups. The inability to rank participants' EI using questionnaires is a concern since FFQ are often used in epidemiological studies with the aim to investigate diet-disease relationships. However, it is possible that we would have been able to find significant correlations if the sample had been larger.

We are not aware of any studies that have evaluated FFQ in pregnant women against TEE measured with the DLW method. However, in a study by Brantsaeter et al., a 255-item FFQ assessing EI among pregnant women was able to capture on average $96 \%$ of the TEE calculated from resting energy expenditure and physical activity measured by a motion sensor, but was not able to rank participants according to $\mathrm{EI}^{(30)}$. We recognize the problems of evaluating assessed EI against the DLW method in pregnant women, who are in a positive energy balance. The extra energy required in pregnancy has been estimated to be $1200 \mathrm{~kJ} / \mathrm{d}$ in the second trimester, for women with a mean gestational weight gain of $12 \cdot 0 \mathrm{~kg}^{(31)}$. However, this does not take into account the decreased physical activity during mid- to late pregnancy. In the present study, EI assessed with the SDQ was on average $862 \mathrm{~kJ} / \mathrm{d}$ higher in the pregnant women compared with the non-pregnant normal-weight women.

More comprehensive questionnaires presumably ought to result in a more complete assessment of intakes, because of their greater level of detail, but this was not the case in the present study. Examples of food items included in the FFQ that are not present as separate food items in the SDQ are eggs, cereals, beans and cooking oil; however, the extra items in the FFQ did not compensate for the lower estimated intakes of the foods/ food groups that were included in both the FFQ and the SDQ when it comes to EI. A possible reason why the SDQ captured as much of the intake as the more comprehensive FFQ is that the design of the questions in the SDQ made it easier for the participants to more accurately report their dietary intake, even though it comprised fewer food items. A previous study showed that the design of the FFQ is more important than its length when it comes to response rate and data quality ${ }^{(32)}$. Furthermore, using a very long questionnaire can result in an overestimation of intake ${ }^{(33)}$ or careless or incomplete questionnaire responses. Subar et al. have shown that using a longer questionnaire does not correspond with a more accurate estimation of $\mathrm{EI}^{(26)}$. However, other studies have shown that the strength of the correlations for nutrient intakes between reference methods is positively related to the length of the questionnaire ${ }^{(13,14)}$. To include all foods eaten is obviously not feasible and there must be a trade-off between the level of detail and feasibility of the questionnaire. The intakes of two food groups were lower when assessed with the SDQ than the FFQ, i.e. snacks and high-fat milk products. The low correlation and the fact that the FFQ captured more of the intake of these food items suggest that the SDQ could be improved with regard to these foods.

The order of the questionnaires could possibly have affected the results of the present study; the participants could be assumed to be able to fill out the second questionnaire better because of learning or less well because of fatigue. However, the questionnaires were completed at least $10 \mathrm{~d}$ apart, which makes it less likely that the completion of the two questionnaires affected each other. Furthermore, the time frame of the SDQ, which goes three months back in time, could have better reflected the current diet and have made it easier for the participants to be able to accurately recall their food intake compared with the FFQ, which asks for the intake over the last twelve months.

A limitation to the present study is that there was no reference method used other than the FFQ with which to compare the intakes of foods and nutrients assessed with the SDQ. A comparison with e.g. $24 \mathrm{~h}$ recalls or weighed food intakes would have been more suitable to determine the relative validity of the SDQ regarding food and nutrient intakes. Another limitation is the seasonal differences and real changes in intakes that could have occurred between the different time periods covered by the SDQ and the FFQ; however, most seasonal foods are not included in the questionnaires. Furthermore, different foods chosen to represent common food groups in the two questionnaires could have biased the comparative results. Moreover, the SDQ and the FFQ do not use the same estimation of portion size, although both questionnaires used weight tables from the National Food Agency to estimate some of the foods ${ }^{(20)}$. Both questionnaires used the Swedish Food Database for calculation of energy and nutrients; however, they did not use the same version of the database for the calculation of macronutrients. A limitation to the SDQ is that it is based on foods common in a traditional Swedish diet, and for this reason a decision was made to exclude vegetarians from the main analysis. However, a sensitivity analysis was performed with the vegetarians included and the underestimation of EI was then $31 \%$ (from 30\%) in the non-pregnant women and $22 \%$ (from $21 \%$ ) in the pregnant women when compared with TEE. Furthermore, the correlation between $\mathrm{EI}_{\mathrm{SDQ}}$ and TEE in the overweight/ obese was reduced from 0.37 to 0.28 when one vegetarian was included. Although it was encouraging that the results were only slightly weaker when including vegetarians, these findings suggest that the SDQ method should be further adapted before using in samples where vegetarianism is common.

A strength of the present study is the use of the DLW method to validate assessed EI in all ninety participants. An advantage of the SDQ is that it is based on intakes from a national dietary survey ${ }^{(19)}$ and thus the questionnaire is suitable for assessment of a mixed Swedish diet. It is therefore likely that the items included in the 
SDQ will make up a considerable part of the respondents' diet. The SDQ could possibly be used in countries with similar dietary culture as the Swedish. The design of the SDQ could also form the basis for new questionnaires that aim to assess dietary intake in other countries or focus on intakes of certain foods or nutrients. Furthermore, the SDQ was designed to be used on one single A4 paper sheet, which can be advantageous in some studies, e.g. in field studies with limited computer and Internet facilities. A potential application of the SDQ is when time is limited but the majority of the dietary intake is still of interest. It can for example be used as a rapid assessment tool in clinical settings. However, the underestimation of EI may limit its applications in studies and clinical settings where more precise information of intake at the individual level is needed. Due to the findings of the present study, future studies validating the SDQ against $24 \mathrm{~h}$ recalls/food diaries or nutrient biomarkers would further clarify its ability to estimate intake of different foods and nutrients. Further studies are also needed before the SDQ can be reliably used in demographic groups other than young pregnant and non-pregnant women (e.g. men and elderly people).

\section{Conclusion}

The SDQ, carefully designed with the aim to make it easier and more time efficient to report dietary intake, underestimated EI of non-pregnant and pregnant, overweight and obese women on a group level but was able to rank the overweight/obese women according to EI. Furthermore, the short questionnaire captured as much or more of the energy, nutrient and food intakes of non-pregnant normal-weight and overweight/ obese women on a group level as a traditional, more extensive FFQ.

\section{Acknowledgements}

Sources of funding: The study was a preparatory project for the LifeGene Study (www.lifegene.se) and was funded by Torsten \& Ragnar Söderberg's Foundation (via LifeGene), Fredrik and Ingrid Thuring's Foundation, and Västerbotten regional health authority (grants to P.W.F.). The funders had no role in the design, analysis or writing of this article. Conflicts of interest: The authors declare that they have no conflicts of interest with respect to this research. Authors' contributions: P.W.F., F.R. and C.L. designed the study and C.L. developed the SDQ; F.R. and P.W.F. collected data; C.L. and L.L. co-supervised the data analysis and interpretation of results; L.B. conducted the DLW analysis; and A.S. conducted data analysis and drafted the manuscript. All authors read and helped revise the manuscript and approved of the final version. Acknowledgements: The authors would like to thank the participants in this study, A. Wright for assistance with DLW analysis and M. Holmgren for assistance with data collection.

\section{Supplementary material}

To view supplementary material for this article, please visit http://dx.doi.org/10.1017/S1368980013003042

\section{References}

1. Rothman KJ, Greenland S \& Lash TL (2008) Modern Epidemilogy, 3d ed. Philadelphia: Lippincott Williams \& Wilkins.

2. Tucker KL (2007) Assessment of usual dietary intake in population studies of gene-diet interaction. Nutr Metab Cardiovasc Dis 17, 74-81.

3. Block G, Hartman AM, Dresser CM et al. (1986) A databased approach to diet questionnaire design and testing. Am J Epidemiol 124, 453-469.

4. Westerterp KR \& Goris AHC (2002) Validity of the assessment of dietary intake: problems of misreporting. Curr Opin Clin Nutr Metab Care 5, 489-493.

5. Andersen LF, Tomten H, Haggarty P et al. (2003) Validation of energy intake estimated from a food frequency questionnaire: a doubly labelled water study. Eur J Clin Nutr 57, 279-284.

6. Scagliusi FB, Ferriolli E, Pfrimer K et al. (2008) Underreporting of energy intake in Brazilian women varies according to dietary assessment: a cross-sectional study using doubly labeled water. J Am Diet Assoc 108, 2031-2040.

7. Prentice AM, Black AE, Coward WA et al. (1986) High levels of energy expenditure in obese women. Br Med J (Clin Res Ed) 292, 983-987.

8. Lafay L, Basdevant A, Charles MA et al. (1997) Determinants and nature of dietary underreporting in a free-living population: the Fleurbaix Laventie Ville Santé (FLVS) Study. Int J Obes Relat Metab Disord 21, 567-573.

9. Weber JL, Reid PM, Greaves KA et al. (2001) Validity of selfreported energy intake in lean and obese young women, using two nutrient databases, compared with total energy expenditure assessed by doubly labeled water. Eur J Clin Nutr 55, 940-950.

10. Lissner L, Troiano RP, Midthune D et al. (2007) OPEN about obesity: recovery biomarkers, dietary reporting errors and BMI. Int J Obes (Lond) 31, 956-961.

11. Cade JE, Burley VJ, Warm DL et al. (2004) Food-frequency questionnaires: a review of their design, validation and utilisation. Nutr Res Rev 17, 5-22.

12. Gibson RS (2005) Principles of Nutritional Assessment, 2nd ed. New York: Oxford University Press.

13. Block G, Hartman AM \& Naughton D (1990) A reduced dietary questionnaire: development and validation. Epidemiology 1, 58-64.

14. Molag ML, de Vries JH, Ocké MC et al. (2007) Design characteristics of food frequency questionnaires in relation to their validity. Am J Epidemiol 166, 1468-1478.

15. Wolk A, Ljung H, Vessby B et al. (1998) Effect of additional questions about fat on the validity of fat estimates from a food frequency questionnaire. Study Group of MRS SWEA. Eur J Clin Nutr 52, 186-192.

16. Brage S, Brage N, Ekelund U et al. (2006) Effect of combined movement and heart rate monitor placement on physical activity estimates during treadmill locomotion and free-living. Eur J Appl Physiol 96, 517-524.

17. Pomeroy J, Brage S, Curtis J et al. (2011) Between-monitor differences in step counts are related to body size: 
implications for objective physical activity measurement. PloS One 27, e18942.

18. World Health Organization (1995) Physical Status: The Use and Interpretation of Anthropometry. Report of a WHO Expert Committee. WHO Technical Report Series no. 854. Geneva: WHO.

19. Becker W \& Pearson M (2002) Riksmaten 1997-98. Dietary habits and nutrient intake in Sweden 1997-98. The second national food consumption survey. Uppsala: Livsmedelsverket (in Swedish).

20. Livsmedelsverket (1999) Weight Tables. Uppsala: Livsmedelsverkets repro (in Swedish).

21. Johansson I, Hallmans G, Wikman A et al. (2002) Validation and calibration of food-frequency questionnaire measurements in the Northern Sweden Health and Disease cohort. Public Health Nutr 5, 487-496.

22. Winkvist A, Hörnell A, Hallmans G et al. (2009) More distinct food intake patterns among women than men in northern Sweden: a population-based survey. Nutr J 8, 12.

23. Johansson G, Wikman A, Ahrén AM et al. (2001) Underreporting of energy intake in repeated 24-hour recalls related to gender, age, weight status, day of interview, educational level, reported food intake, smoking habits and area of living. Public Health Nutr 4, 919-927.

24. Bluck LJC (2008) Doubly labelled water for the measurement of total energy expenditure in man - progress and applications in the last decade. Nutr Bull 33, 80-90.

25. Livingstone MBE \& Black AE (2003) Markers of the validity of reported energy intake. J Nutr 133, Suppl. 3, 895S-920S.

26. Subar AF, Kipnis V, Troiano RP et al. (2003) Using intake biomarkers to evaluate the extent of dietary misreporting in a large sample of adults: the OPEN study. Am J Epidemiol 158, $1-13$.

27. Kroke A, Klipstein-Grobusch K, Voss S et al. (1999) Validation of a self-administered food-frequency questionnaire administered in the European Prospective Investigation into Cancer and Nutrition (EPIC) Study: comparison of energy, protein, and macronutrient intakes estimated with the doubly labeled water, urinary nitrogen, and repeated 24-h dietary recall methods. Am J Clin Nutr 70, 439-447.

28. Lutomski JE, van den Broeck J, Harrington J et al. (2011) Sociodemographic, lifestyle, mental health and dietary factors associated with direction of misreporting of energy intake. Public Health Nutr 14, 532-541.

29. Yannakoulia M, Panagiotakos DB, Pitsavos C et al. (2007) Low energy reporting related to lifestyle, clinical, and psychosocial factors in a randomly selected population sample of Greek adults: the ATTICA Study. J Am Coll Nutr 26, 327-333.

30. Brantsaeter AL, Haugen M, Alexander J et al. (2008) Validity of a new food frequency questionnaire for pregnant women in the Norwegian Mother and Child Cohort Study (MoBa). Matern Child Nutr 4, 28-43.

31. Butte NF \& King JC (2005) Energy requirements during pregnancy and lactation. Public Health Nutr 8, 1010-1027.

32. Subar AF, Ziegler RG, Thompson FE et al. (2001) Is shorter always better? Relative importance of questionnaire length and cognitive ease on response rates and data quality for two dietary questionnaires. Am J Epidemiol 153, 404-409.

33. Krebs-Smith SM, Heimendinger J, Subar AF et al. (1994) Estimating fruit and vegetable intake using food frequency questionnaires: a comparison of instruments. Am J Clin Nutr 59, 283 S. 